\title{
GEODESICS ON EXTENSIONS OF THE LIE ALGEBRA OF VECTOR FIELDS ON THE CIRCLE
}

\author{
CORNELIA VIZMAN \\ Department of Mathematics, West University of Timişoara \\ Bd. V. Parvan 4, 1900 Timişoara, Romania \\ E-mail:vizman@math.uvt.ro
}

\begin{abstract}
We consider Lie algebra 2-cocycles on the Lie algebra of vector fields on the circle with values in the space of tensor densities on the circle, whose integral over the circle is the Virasoro cocycle. Geodesic equations on the abelian extensions defined by these cocycles are determined.
\end{abstract}

1. Introduction. Some partial differential equations can be written as geodesic equations on groups of diffeomorphisms. Considering right invariant $L^{2}$ or $H^{1}$ metrics on the group of diffeomorphisms of the circle and on the group of volume preserving diffeomorphisms of a Riemannian manifold $M$, as well as abelian extensions of these groups, the following geodesic equations are obtained: Euler equation of motion of a perfect fluid, averaged Euler equation, equations of ideal magneto-hydrodynamics, Burger's equation, Korteweg-de-Vries equation, Camassa-Holm shallow water equation, superconductivity equation and equation of motion of a charged ideal fluid.

By the method of transferring the problem of studying an equation to the problem of finding geodesics on a group of diffeomorphisms, it is possible to give more information on this equation: to say something about the stability of the partial differential equation by determining the sign of the curvature of the diffeomorphism group, to get local well posedness of the Cauchy problem for the partial differential equation as well as smooth dependence on the initial data or to indicate the existence of stable perturbations by determining the conjugate points of geodesics.

The Riemannian geometry of Lie groups with right invariant metrics lives actually on the level of their Lie algebras: the geodesic equation can be written in terms of the right logarithmic derivative of the curve, the Riemannian tensor is determined by its values at

2000 Mathematics Subject Classification: 17B56, 35Q53.

Key words and phrases: abelian extensions, Virasoro cocycle, geodesics.

Received 23 March 2001; revised 21 July 2001.

The paper is in final form and no version of it will be published elsewhere. 
the identity. So, by an abuse of language, we can speak about geodesic equations on Lie algebras with inner products.

The geodesic equation for the right invariant $L^{2}$ metric on the Virasoro-Bott group, the 1-dimensional central extension of the group of diffeomorphisms on the circle $\operatorname{Diff}_{+}\left(S^{1}\right)$ by Thurston-Bott group cocycle $c_{0}(\varphi, \psi)=\int_{S^{1}} \log \left(\varphi^{\prime} \circ \psi\right) d \log \psi^{\prime}$, is the Korteweg-de-Vries equation $u_{t}+3 u u^{\prime}+a u^{\prime \prime \prime}=0[\mathrm{OK}]$. Here the prime stands for $\frac{\partial}{\partial x}$ and the index $t$ for $\frac{\partial}{\partial t}$. The corresponding real valued Lie algebra cocycle on $\mathfrak{X}\left(S^{1}\right)$, the Lie algebra of vector fields on the circle, is the Virasoro cocycle:

$$
\operatorname{vir}(X, Y)=\int_{S^{1}}\left(X^{\prime} Y^{\prime \prime}-X^{\prime \prime} Y^{\prime}\right) d x=\int_{S^{1}}\left(X^{\prime \prime \prime} Y-X Y^{\prime \prime \prime}\right) d x
$$

and its cohomology class is a basis for $H^{2}\left(\mathfrak{X}\left(S^{1}\right)\right)$, the second Lie algebra cohomology group. Do the expressions under the integral in (1) define Lie algebra cocycles with values in smooth functions on $S^{1}$ ?

A similar setting is the following: every closed differential 2-form on a compact manifold $M$ determines a 2-cocycle on $\mathfrak{X}(M)$ with values in $C^{\infty}(M)$, so it defines an abelian extension of the Lie algebra of vector fields by the module of smooth functions. When a Riemannian metric on $M$ is given, the integral of this cocycle over $M$ gives a real valued 2-cocycle on the Lie algebra of divergence free vector fields on $M$, called the Lichnerowicz cocycle, so it defines a 1-dimensional central extension of this Lie algebra. Considering $L^{2}$ inner products, the geodesic equation on this central extension of the Lie algebra of divergence free vector fields on $M$ is the superconductivity equation $[\mathrm{Z}][\mathrm{R}]$ and the geodesic equations on the abelian extension above (restricted to the Lie algebra of divergence free vector fields) is the equation of motion of a charged ideal fluid [V].

Let $\mathfrak{F}_{n}$ be the space of $n$-densities on the circle. The canonical action of the Lie algebra $\mathfrak{X}\left(S^{1}\right)$ on $\mathfrak{F}_{n}$ is $b(X) f=-X f^{\prime}-n X^{\prime} f$. Only in this context the expressions under the integral in (1) become Lie algebra cocycles. For $n=1$ we get the action on 1 -forms and the expression $\omega(X, Y)=X^{\prime} Y^{\prime \prime}-X^{\prime \prime} Y^{\prime}$ defines a 2-cocycle on $\mathfrak{X}\left(S^{1}\right)$ with values in $\Omega^{1}(M)$. For $n=2$ we get the action on quadratic differentials (the coadjoint action in the group of diffeomorphisms on the circle) and $\rho(X, Y)=X^{\prime \prime \prime} Y-X Y^{\prime \prime \prime}$ is a cocycle on $\mathfrak{X}\left(S^{1}\right)$ with values in $Q\left(S^{1}\right)$.

A theorem in $[\mathrm{F}]$ determines the dimension of the cohomology groups of $\mathfrak{X}\left(S^{1}\right)$ with values in tensor densities. It follows that the dimension of $H^{2}\left(\mathfrak{X}\left(S^{1}\right) ; \mathfrak{F}_{n}\right)$ is 2 for $n=0,1,2$. In $[\mathrm{OR}]$ extensions of the group $\operatorname{Diff}_{+}\left(S^{1}\right)$ by the module of tensor densities are considered and in $[\mathrm{K}]$ coadjoint orbits on these extensions are studied. The dimension of the group $H_{c}^{2}\left(\operatorname{Diff}_{+}\left(S^{1}\right) ; \mathfrak{F}_{n}\right)$ of differentiable cohomology, which classifies these extensions, is 1 for $n=0,1,2$. We write down the geodesic equations for the corresponding group extensions with $L^{2}$ invariant metrics, as well as for the Lie algebra extensions by cocycles which can not be integrated to a group cocycle with $L^{2}$ inner products.

2. Right invariant metrics on Lie groups. Let $G$ be a regular Lie group in the sense of Kriegl-Michor [KM], with Lie algebra $\mathfrak{g}$. Let $\rho_{x}$ be the right translation by $x \in G$. Any right invariant Riemannian metric on $G$ is determined by its value at the identity $\langle\rangle:, \mathfrak{g} \times \mathfrak{g} \rightarrow \mathbb{R}$, i.e. by a positive definite bounded inner product on $\mathfrak{g}$. Let 
$g: I=[a, b] \rightarrow G$ be a smooth curve and $u: I \rightarrow \mathfrak{g}$ its right logarithmic derivative (the velocity field in the right trivialization) $u(t)=T \rho_{g(t)^{-1}} \cdot g^{\prime}(t)$. In terms of $u$ the geodesic equation for $g$ has the expression [MR]

$$
u_{t}=-\operatorname{ad}(u)^{\top} u,
$$

where $u_{t}$ means time derivative of $u$ and, for $X \in \mathfrak{g}, \operatorname{ad}(X)^{\top}$ is the adjoint of $\operatorname{ad}(X)$ : $\mathfrak{g} \rightarrow \mathfrak{g}$ with respect to $\langle$,$\rangle (if this adjoint does exist). Equation (2) is the Euler-Poincaré$ equation for right invariant systems, where the kinetic energy is the Lagrangian. By abuse of language we will call equation (2) the geodesic equation on the Lie algebra $\mathfrak{g}$ with inner product $\langle$,$\rangle , even if a corresponding Lie group G$ does not exist.

EXAMPLE 1. Let $G=\operatorname{Diff}_{+} S^{1}$ be the group of orientation preserving diffeomorphisms of the circle and $\mathfrak{g}=\mathfrak{X}\left(S^{1}\right)$ the Lie algebra of vector fields. The Lie bracket is $[X, Y]=$ $X^{\prime} Y-X Y^{\prime}$, the negative of the usual bracket on vector fields (vector fields on the circle are identified here with their coefficient function). We consider the right invariant metric on $G$ given by the $L^{2}$ inner product $\langle X, Y\rangle=\int_{S^{1}} X Y d x$. The transpose of $\operatorname{ad}(X)$ is $\operatorname{ad}(X)^{\top} Y=2 X^{\prime} Y+X Y^{\prime}$, because:

$$
\begin{aligned}
\left\langle\operatorname{ad}(X)^{\top} Y, Z\right\rangle & =\langle Y, \operatorname{ad}(X) Z\rangle=\int_{S^{1}} Y\left(X^{\prime} Z-X Z^{\prime}\right) d x \\
& =\int_{S^{1}}\left(X^{\prime} Y+(X Y)^{\prime}\right) Z d x=\left\langle 2 X^{\prime} Y+X Y^{\prime}, Z\right\rangle .
\end{aligned}
$$

It follows that the geodesic equation in terms of the right logarithmic derivative $u: I \rightarrow$ $\mathfrak{X}\left(S^{1}\right)$ is Burger's equation:

$$
u_{t}=-3 u u^{\prime}
$$

ExAmPLE 2. Let $G=\operatorname{Diff}_{v o l}(M)$ be the group of volume preserving diffeomorphisms of a compact Riemannian manifold $(M, g)$ with induced volume form $\mu$. Let $\mathfrak{g}=\mathfrak{X}_{\text {vol }}(M)$ be the Lie algebra of divergence free vector fields. We consider the right invariant metric on $G$ given by the $L^{2}$ inner product $\langle X, Y\rangle=\int_{M} g(X, Y) \mu$. The transpose of $\operatorname{ad}(X)$ is $\operatorname{ad}(X)^{\top} Y=P\left(\nabla_{X} Y+(\nabla X)^{\top} Y\right)$ where $P$ denotes the orthogonal projection on the space of divergence free vector fields and $\nabla$ the Levi Civita covariant derivative. Then the geodesic equation in terms of the right logarithmic derivative $u: I \rightarrow \mathfrak{X}_{\text {vol }}(M)$ is the Euler equation for the ideal flow

$$
u_{t}=-\nabla_{u} u-\operatorname{grad} p, \quad \operatorname{div} u=0 .
$$

3. Extensions of Lie algebras. Let $b$ be a Lie algebra action of $\mathfrak{g}$ on the vector space $V$. The cohomology groups of the Lie algebra $\mathfrak{g}$ with values in the $\mathfrak{g}$-module $V$, denoted $H^{k}(\mathfrak{g} ; V)$, are defined by the following derivation on multilinear skew-symmetric mappings from $\mathfrak{g} \times \ldots \times \mathfrak{g}$ to $V$ :

$$
\begin{aligned}
d \omega\left(X_{1}, \ldots, X_{k+1}\right)= & \sum_{i=1}^{k+1}(-1)^{i} b\left(X_{i}\right) \omega\left(X_{1}, \ldots, \hat{X}_{i}, \ldots, X_{k+1}\right) \\
& +\sum_{1 \leq i<j \leq k+1}(-1)^{i+j-1} \omega\left(\left[X_{i}, X_{j}\right], X_{1}, \ldots, \hat{X}_{i}, \ldots, \hat{X}_{j}, \ldots, X_{k+1}\right) .
\end{aligned}
$$


In particular a 2-cocycle satisfies the relation

$$
\sum_{c y c l} \omega\left(\left[X_{1}, X_{2}\right], X_{3}\right)=\sum_{c y c l} b\left(X_{1}\right) \omega\left(X_{2}, X_{3}\right)
$$

and it determines an abelian Lie algebra extension $\mathfrak{e}$ of $\mathfrak{g}$ by $V$ with Lie bracket

$$
\left[\left(X_{1}, f_{1}\right),\left(X_{2}, f_{2}\right)\right]=\left(\left[X_{1}, X_{2}\right], b\left(X_{1}\right) f_{2}-b\left(X_{2}\right) f_{1}+\omega\left(X_{1}, X_{2}\right)\right) .
$$

When the $\mathfrak{g}$-module $V$ is trivial, i.e. $b=0$, we get a central extension, when $\omega=0$ we get the semidirect product $\mathfrak{g} \ltimes V$. There is a 1-1 correspondence between the second cohomology group $H^{2}(\mathfrak{g} ; V)$ and equivalence classes of abelian extensions $\mathfrak{e}$ of $\mathfrak{g}$ by $V$ :

$$
0 \rightarrow V \rightarrow \mathfrak{e} \rightarrow \mathfrak{g} \rightarrow 0 .
$$

Following $[\mathrm{V}]$ we write down the geodesic equations on $\mathfrak{e}$ with respect to the inner product

$$
\left\langle\left(X_{1}, f_{1}\right),\left(X_{2}, f_{2}\right)\right\rangle_{\mathfrak{e}}=\left\langle X_{1}, X_{2}\right\rangle_{\mathfrak{g}}+\left\langle f_{1}, f_{2}\right\rangle_{V}
$$

where $\langle,\rangle_{\mathfrak{g}}$ and $\langle,\rangle_{V}$ are inner products on $\mathfrak{g}$ and $V$ such that the transpose $\operatorname{ad}(X)^{\top}$ : $\mathfrak{g} \rightarrow \mathfrak{g}$ exists for any $X \in \mathfrak{g}$. We suppose also that the transpose $b(X)^{\top}: V \rightarrow V$ exists for any $X \in \mathfrak{g}$ and there exist maps $h: V \rightarrow L(\mathfrak{g})$ linear (actually $h$ takes values in the space of skew-adjoint operators on $\mathfrak{g})$ and $l: V \times V \rightarrow \mathfrak{g}$ bilinear, defined by the relations

$$
\left\langle\omega\left(X_{1}, X_{2}\right), f\right\rangle_{V}=\left\langle h(f) X_{1}, X_{2}\right\rangle_{\mathfrak{g}}, \quad\left\langle b(X) f_{1}, f_{2}\right\rangle_{V}=\left\langle l\left(f_{1}, f_{2}\right), X\right\rangle_{\mathfrak{g}} .
$$

Proposition 1. With the conditions above, the geodesic equation on the abelian extension $\mathfrak{e}$ for $u: I \rightarrow \mathfrak{g}$ and $f: I \rightarrow V$ is:

$$
u_{t}=-\operatorname{ad}(u)^{\top} u-h(f) u+l(f, f), \quad f_{t}=-b(u)^{\top} f .
$$

In the special case of central extensions, the geodesic equation becomes

$$
u_{t}=-\operatorname{ad}(u)^{\top} u-h(a) u, \quad a \in V .
$$

ExAmple $3[\mathrm{OK}]$. The Korteweg-de-Vries equation is a geodesic equation on the Virasoro Lie algebra. Let $\mathfrak{g}=\mathfrak{X}\left(S^{1}\right)$ with $L^{2}$ inner product, $V=\mathbb{R}$ and $\omega(X, Y)=$ $\operatorname{vir}(X, Y)=2 \int_{S^{1}} X^{\prime} Y^{\prime \prime} d x$. Observe that $\omega(X, Y)=2 \int_{S^{1}} X^{\prime \prime \prime} Y d x$, so $h(a) X=2 a X^{\prime \prime \prime}$ for $a \in \mathbb{R}$ and the geodesic equation (4) is the $\mathrm{KdV}$ equation (see also example 1 ):

$$
u_{t}=-3 u u^{\prime}-2 a u^{\prime \prime \prime}, \quad a \in \mathbb{R} .
$$

ExAMPLE $4[\mathrm{R}][\mathrm{Z}]$. The superconductivity equation is a geodesic equation on a central extension of the Lie algebra of divergence free vector fields $\mathfrak{X}_{\text {vol }}(M)$ with the Lichnerowicz cocycle $\omega(X, Y)=\int_{M} \eta(X, Y) \mu$. Here $M$ is a compact 3 dimensional Riemannian manifold and $\eta$ is a closed differential 2-form on $M$, hence the vector field $B$ defined by $i_{B} \mu=-\eta$ is divergence free (the magnetic field). When considering the $L^{2}$ inner product on $\mathfrak{g}=\mathfrak{X}_{\text {vol }}(M)$, we can write $\omega(X, Y)=\langle X \times B, Y\rangle$, where $\times$ denotes the cross product determined by $\mu$, i.e. $X \times Y=\left(i_{X \wedge Y} \mu\right)^{\sharp}$. Then $h(a) X=a P(X \times B)$ and, with the notations from example 2 , we get the superconductivity equation as geodesic equation:

$$
u_{t}=-\nabla_{u} u-a u \times B-\operatorname{grad} p, \quad a \in \mathbb{R} .
$$

EXAmple $5[\mathrm{~V}]$. The equation of motion of an ideal charged fluid in a magnetic field is a geodesic equation on the abelian extension of $\mathfrak{g}=\mathfrak{X}_{\text {vol }}(M)$ by $V=C^{\infty}(M)$ with 
cocycle $\omega(X, Y)=\eta(X, Y)$, where $\eta$ is again a closed 2-form on the 3 dimensional compact Riemannian manifold $M$ with corresponding divergence free vector field $B$ (magnetic field). With $L^{2}$ inner products on $\mathfrak{g}$ and $V$, the action $b(X) f=-d f . X$ of $\mathfrak{g}$ on $V$ is skewadjoint, which implies $l(f, f)=0$, and $\langle\eta(X, Y), f\rangle_{V}=\langle f X \times B, Y\rangle_{\mathfrak{g}}$, which implies $h(f) X=P(f X \times B)$. The geodesic equation (3) for $u: I \rightarrow \mathfrak{X}_{v o l}(M)$ (velocity field) and $f: I \rightarrow C^{\infty}(M)$ (charge density) is the equation of motion of an ideal charged fluid in a magnetic field

$$
u_{t}=-\nabla_{u} u-f u \times B-\operatorname{grad} p, \quad f_{t}=-d f . u .
$$

4. Tensor densities on the circle. The Lichnerowicz cocycle in example 4 is the integral over $M$ of the function valued cocycle in example 5. The Virasoro cocycle in example 3 is the integral over the circle of the expressions: $X^{\prime} Y^{\prime \prime}-X^{\prime \prime} Y^{\prime}$ or $X Y^{\prime \prime \prime}-X^{\prime \prime \prime} Y$. These do not define cocycles with values in the module of functions on the circle, but do define cocycles with values in tensor densities on the circle.

Let $n \in \mathbb{Z}$. The space of $n$-densities on the circle is

$$
\mathfrak{F}_{n}=\left\{\begin{array}{rr}
C^{\infty}\left(\otimes^{n} T^{*} S^{1}\right) & \text { for } n>0 \\
C^{\infty}\left(\otimes^{-n} T S^{1}\right) & \text { for } n<0 \\
C^{\infty}\left(S^{1}\right) & \text { for } n=0
\end{array}\right.
$$

When no confusion is possible, we represent densities by their coefficient functions. The Lie group $\operatorname{Diff}_{+}\left(S^{1}\right)$ acts on $\mathfrak{F}_{n}$ by

$$
B(\varphi) f=\left(f \circ \varphi^{-1}\right)\left(\varphi^{\prime} \circ \varphi^{-1}\right)^{-n}
$$

and the corresponding Lie algebra action of $\mathfrak{X}\left(S^{1}\right)$ is

$$
b(X) f=-X f^{\prime}-n X^{\prime} f .
$$

For $n=-1$ we get the adjoint action on vector fields, for $n=0$ the action on functions by derivations, for $n=1$ the negative of the Lie derivative on 1-forms and for $n=2$ the coadjoint action on the space $Q\left(S^{1}\right)$ of quadratic differentials. We can consider also the module $\mathfrak{F}_{\lambda}$ of $\lambda$ densities, for every $\lambda \in \mathbb{R}$.

The following theorem $[\mathrm{F}]$ determines the dimension of all cohomology groups of $\mathfrak{X}\left(S^{1}\right)$ with values in tensor densities on the circle for $n \geq 0$.

TheOREM 2. Let $n=\frac{3 r^{2} \pm r}{2}$. Then

$$
H^{q}\left(\mathfrak{X}\left(S^{1}\right) ; \mathfrak{F}_{n}\right)=H^{q-r}\left(S^{1} \times S^{1} \times \Omega S^{3} ; \mathbb{R}\right),
$$

where $\Omega$ denotes the loop space.

Moreover $H^{*}\left(\mathfrak{X}\left(S^{1}\right) ; \mathfrak{F}_{n}\right)$ is a free $H^{*}\left(\mathfrak{X}\left(S^{1}\right) ; C^{\infty}\left(S^{1}\right)\right)$-module with one generator, which belongs to $H^{r}\left(\mathfrak{X}\left(S^{1}\right) ; \mathfrak{F}_{n}\right)$.

As a consequence we get that $\operatorname{dim} H^{2}\left(\mathfrak{X}\left(S^{1}\right) ; \mathfrak{F}_{n}\right)=2$ for $n=0,1,2$. In these cases the generators of $H^{*}\left(\mathfrak{X}\left(S^{1}\right) ; \mathfrak{F}_{n}\right)$ are explicitly determined in $[\mathrm{F}]$ :

Proposition 3. The generators of the ring $H^{*}\left(\mathfrak{X}\left(S^{1}\right) ; C^{\infty}\left(S^{1}\right)\right)$ are represented by the cocycles $\alpha_{0}(X)=X^{\prime}, \beta_{0}(X)=X$ and the Virasoro cocycle $\operatorname{vir}(X, Y)=2 \int_{S^{1}} X^{\prime} Y^{\prime \prime} d x$ $\in \mathbb{R} \subset C^{\infty}\left(S^{1}\right)$. 
$H^{*}\left(\mathfrak{X}\left(S^{1}\right) ; \Omega^{1}\left(S^{1}\right)\right)$ is a free $H^{*}\left(\mathfrak{X}\left(S^{1}\right) ; C^{\infty}\left(S^{1}\right)\right)$-module with one one-dimensional generator, represented by the cocycle $\alpha_{1}(X)=X^{\prime \prime}$.

$H^{*}\left(\mathfrak{X}\left(S^{1}\right) ; Q\left(S^{1}\right)\right)$ is a free $H^{*}\left(\mathfrak{X}\left(S^{1}\right) ; C^{\infty}\left(S^{1}\right)\right)$-module with one one-dimensional generator, represented by the cocycle $\alpha_{2}(X)=X^{\prime \prime \prime}$.

Next we enumerate cocycles representing elements of a basis for each of these second cohomology groups. A basis for $H^{2}\left(\mathfrak{X}\left(S^{1}\right) ; C^{\infty}\left(S^{1}\right)\right)$ is represented by $\sigma(X, Y)=X^{\prime} Y-$ $X Y^{\prime}$ and the Virasoro cocycle $\operatorname{vir}(X, Y)=2 \int_{S^{1}} X^{\prime} Y^{\prime \prime} d x \in \mathbb{R} \subset C^{\infty}\left(S^{1}\right)$. A basis for $H^{2}\left(\mathfrak{X}\left(S^{1}\right) ; \Omega^{1}\left(S^{1}\right)\right)$ is represented by the cocycles

$$
\tau(X, Y)=X Y^{\prime \prime}-X^{\prime \prime} Y, \quad \omega(X, Y)=X^{\prime} Y^{\prime \prime}-X^{\prime \prime} Y^{\prime} .
$$

A basis for $H^{2}\left(\mathfrak{X}\left(S^{1}\right) ; Q\left(S^{1}\right)\right)$ is represented by the cocycles

$$
\rho(X, Y)=X^{\prime \prime \prime} Y-X Y^{\prime \prime \prime}, \quad \eta(X, Y)=X^{\prime \prime \prime} Y^{\prime}-X^{\prime} Y^{\prime \prime \prime} .
$$

By taking the integral of $\omega$ and $\rho$ over the circle we obtain the Virasoro cocycle. For all $n \neq 1$ the cocycle $\omega(X, Y)=\left(X^{\prime} Y^{\prime \prime}-X^{\prime \prime} Y^{\prime}\right) d x^{n}$ is exact, namely $\omega=d \gamma$ with $\gamma(X)=X^{\prime \prime} d x^{n}$. On the other hand, $\rho(X, Y)=\left(X^{\prime \prime \prime} Y-X Y^{\prime \prime \prime}\right) d x^{n}$ is not a cocycle for $n \neq 2$ and vir is not a cocycle for $n \neq 0$. The integral of $\tau$ and $\eta$ over the circle gives zero.

The group $H_{c}^{2}\left(\operatorname{Diff}_{+}\left(S^{1}\right) ; \mathfrak{F}_{\lambda}\right)$ of differentiable group cohomology classifies extensions of Diff $+\left(S^{1}\right)$ by the module $\mathfrak{F}_{\lambda}$ of $\lambda$ densities on the circle. Ovsienko and Roger in [OR] give explicit expressions of cocycles which determine a basis for $H_{c}^{2}\left(\operatorname{Diff}_{+}\left(S^{1}\right) ; \mathfrak{F}_{\lambda}\right)=\mathbb{R}$ for $\lambda=0,1,2,5,7$. These cover all the cases where the group cohomology is non-trivial. Only the Lie algebra cocycles given by differential operators without zero order terms can be integrated to the group. From the list above these are vir, $\omega$ and $\eta$.

The Thurston-Bott cocycle

$$
c_{0}(\varphi, \psi)=\int_{S^{1}} \log \left(\varphi^{\prime} \circ \psi\right) d \log \psi^{\prime}
$$

is a group cocycle integrating the Virasoro cocycle vir. A group cocycle on Diff $+\left(S^{1}\right)$ with values in $\Omega^{1}\left(S^{1}\right)$ integrating $\omega$ is

$$
c_{1}(\varphi, \psi)=\left(\log \left(\psi^{-1}\right)^{\prime} \circ \varphi^{-1}\right) d \log \left(\varphi^{-1}\right)^{\prime} .
$$

A group cocycle with values in quadratic differentials $Q\left(S^{1}\right)$ and integrating $\eta$ is

$$
c_{2}(\varphi, \psi)=\left(\log \left(\psi^{-1}\right)^{\prime} \circ \varphi^{-1}\right) S\left(\varphi^{-1}\right) d x^{2},
$$

where $S$ denotes the Schwarzian derivative $S(\varphi)=\frac{\varphi^{\prime \prime \prime}}{\varphi^{\prime}}-\frac{3}{2}\left(\frac{\varphi^{\prime \prime}}{\varphi^{\prime}}\right)^{2}$.

A first remark is that

$$
\begin{aligned}
& a_{0}(\varphi)=\log \left(\varphi^{-1}\right)^{\prime} \\
& a_{1}(\varphi)=d \log \left(\varphi^{-1}\right)^{\prime} \\
& a_{2}(\varphi)=S\left(\varphi^{-1}\right) d x^{2}
\end{aligned}
$$

are 1 -cocycles on $\operatorname{Diff}_{+}\left(S^{1}\right)$ with values in $\mathfrak{F}_{n}, n=0,1,2$ integrating $\alpha_{0}, \alpha_{1}$ and $\alpha_{2}$ respectively ( $\beta_{0}$ can not be integrated to a group cocycle). A second remark is that $c_{1}$ is a cap product of $a_{0}$ with $a_{1}$ and $c_{2}$ is a cap product of $a_{0}$ with $a_{2}$. The representing cocycles here differ from those in $[\mathrm{OR}]$ because there they consider the $\operatorname{Diff}_{+}\left(S^{1}\right)$ action on $\mathfrak{F}_{n}$ given by $\varphi \cdot f=B\left(\varphi^{-1}\right) f=(f \circ \varphi)\left(\varphi^{\prime}\right)^{n}$. 
5. Geodesic equations. Let $\widetilde{G}$ be the abelian extension of $G=\operatorname{Diff}_{+}\left(S^{1}\right)$ by the module of differential 1-forms on the circle $V=\mathfrak{F}_{1}=\Omega^{1}\left(S^{1}\right)$ defined by the cocycle $c_{1}$ from (6). We consider the right invariant $L^{2}$ metric on $\widetilde{G}$ coming from the inner product $\left\langle\left(X_{1}, f_{1}\right),\left(X_{2}, f_{2}\right)\right\rangle=\int_{S^{1}}\left(X_{1} X_{2}+f_{1} f_{2}\right) d x$ on $\tilde{\mathfrak{g}}$, the abelian extension of $\mathfrak{g}=\mathfrak{X}\left(S^{1}\right)$ by the module $\mathfrak{F}_{1}$ defined by the cocycle $\omega(X, Y)=X^{\prime} Y^{\prime \prime}-X^{\prime \prime} Y^{\prime}$.

In this section we will determine the geodesic equation on $\widetilde{G}$, using again proposition 1. The action of vector fields on 1 -forms is $b(X) f=-X f^{\prime}-X^{\prime} f$ with adjoint $b(X)^{\top} f=X f^{\prime}$. In this case $b(X)$ is not a skew-adjoint operator: the relation $\left\langle b(X) f_{1}, f_{2}\right\rangle_{V}=\left\langle l\left(f_{1}, f_{2}\right), X\right\rangle_{\mathfrak{g}}$ defines the mapping $l\left(f_{1}, f_{2}\right)=f_{1} f_{2}^{\prime}$ with $l(f, f) \neq 0$. What is the mapping $h$ in this case?

$$
\begin{aligned}
\langle h(f) X, Y\rangle & =\langle\omega(X, Y), f\rangle=\int_{S^{1}} f\left(X^{\prime} Y^{\prime \prime}-X^{\prime \prime} Y^{\prime}\right) d x \\
& =\int_{S^{1}} Y\left(\left(f X^{\prime \prime}\right)^{\prime}+\left(f X^{\prime}\right)^{\prime \prime}\right) d x=\left\langle 2 X^{\prime \prime \prime} f+3 X^{\prime \prime} f^{\prime}+X^{\prime} f^{\prime \prime}, Y\right\rangle
\end{aligned}
$$

and we get $h(f) X=2 X^{\prime \prime \prime} f+3 X^{\prime \prime} f^{\prime}+X^{\prime} f^{\prime \prime}$. Then the geodesic equation is

$$
u_{t}=-3 u u^{\prime}-2 u^{\prime \prime \prime} f-3 u^{\prime \prime} f^{\prime}-u^{\prime} f^{\prime \prime}+f f^{\prime}, \quad f_{t}=-u f^{\prime} .
$$

In particular, for the initial condition $f_{0}=a$ constant, we obtain the Kortewegde-Vries equation. This is similar to examples 4 and 5: considering an initial condition with constant $f_{0}$ in the equation of motion of an ideal charged fluid, we obtain the superconductivity equation.

In a similar way geodesic equations corresponding to the other listed cocycles, elements of the basis in $H^{2}\left(\mathfrak{X}\left(S^{1}\right) ; \mathfrak{F}_{n}\right), n=0,1,2$, can be written.

Case $n=0$ : for $\sigma(X, Y)=X^{\prime} Y-X Y^{\prime}$ the geodesic equation is

$$
u_{t}=-3 u u^{\prime}+u f^{\prime}+2 u^{\prime} f-f f^{\prime}, \quad f_{t}=-u f^{\prime}-u^{\prime} f,
$$

and for $\operatorname{vir}(X, Y)=2 \int_{S^{1}} X^{\prime} Y^{\prime \prime} d x$ the geodesic equation is

$$
u_{t}=-3 u u^{\prime}+2 u^{\prime \prime \prime} \int_{S^{1}} f d x-f f^{\prime}, \quad f_{t}=-u f^{\prime}-u^{\prime} f .
$$

Case $n=1$ : for $\tau(X, Y)=X Y^{\prime \prime}-X^{\prime \prime} Y$ the geodesic equation is

$$
u_{t}=-3 u u^{\prime}+2 u^{\prime} f^{\prime}-u f^{\prime \prime}+f f^{\prime}, \quad f_{t}=-u f^{\prime},
$$

Case $n=2$ : for $\rho(X, Y)=X^{\prime \prime \prime} Y-X Y^{\prime \prime \prime}$ the geodesic equation is

$$
u_{t}=-3 u u^{\prime}+u^{\prime \prime \prime} f+(u f)^{\prime \prime \prime}+3 f f^{\prime}, \quad f_{t}=-u f^{\prime}+u^{\prime} f,
$$

and for $\eta(X, Y)=X^{\prime \prime \prime} Y^{\prime}-X^{\prime} Y^{\prime \prime \prime}$ the geodesic equation is

$$
u_{t}=-3 u u^{\prime}+2 u^{\prime \prime \prime} f^{\prime}+3 u^{\prime \prime} f^{\prime \prime}+u^{\prime} f^{\prime \prime \prime}+3 f f^{\prime}, \quad f_{t}=-u f^{\prime}+u^{\prime} f .
$$

\section{References}

[AK] V. I. Arnold and B. A. Khesin, Topological Methods in Hydrodynamics, Springer-Verlag, 1998.

[AMR] D. Alekseevsky, P. W. Michor and W. Ruppert, Extensions of Lie algebras, Preprint ESI 881 (2000), math. DG/0005042. 
[F] D. B. Fuks, Cohomology of Infinite-Dimensional Lie Algebras, Nauka, Moscow. Engl. transl.: Contemp. Sov. Math., Consultants Bureau, New York, 1986.

[K] C. Kapoudjan, Coadjoint orbits of extensions of Diff ${ }^{+}\left(S^{1}\right)$ by modules of tensor densities, J. Geom. Phys. 33 (2000) 1.

[KM] A. Kriegl and P. W. Michor, The Convenient Setting of Global Analysis, Mathematical Surveys and Monographs 53, Amer. Math. Soc., 1997.

[MR] P. W. Michor and T. Ratiu, On the geometry of the Virasoro-Bott group, J. Lie Theory 8 (1998) 293, math. DG/9801115.

[OK] V. Y. Ovsienko and B. A. Khesin, Korteweg-de-Vries superequations as an Euler equation, Funct. Anal. Appl. 21 (1987) 329.

[OR] V. Ovsienko and C. Roger, Generalizations of Virasoro group and Virasoro algebra through extensions by modules of tensor densities on $S^{1}$, Indag. Math. N.S. 9 (1998) 277.

[R] C. Roger, Extensions centrales d'algèbres et de groupes de Lie de dimension infinie, algèbre de Virasoro et généralisations, Rep. Math. Phys. 35 (1995) 225.

[V] C. Vizman, Geodesics on extensions of Lie groups and stability: the superconductivity equation, Phys. Lett. A 284 (2001) 23.

[Z] V. Zeitlin, Vorticity and waves: geometry of phase-space and the problem of normal variables, Phys. Lett. A 164 (1992) 177. 